(Aus dem physiologischen Institut zu Bonn.)

\title{
Ueber die Kjeldahl-Wilfarth'sche Methode der Stickstoffbestimmung unter Berücksichtigung ihrer Anwendung zu Stoffwechselversuchen.
}

Von

Dr. P. Argutinsky aus St. Petersbnrg.

Hierzu 1 Holzschnintt.

Unter den wesentlichen Modificationen der Kjeldahl'schen Methode der Stickstoff bestimmung bezeichnet wobl keine einen so grossen Fortsehritt und hat solch eine grosse praktische Bedentung, wie die von Wilfarth. Bekanntlich hat Wilfarth ${ }^{1}$ ) den glücklichen Griff gethan, die Oxydation mit $\mathrm{H}_{2} \mathrm{SO}_{4}$ durch Zusatz von Metalloxyden zu beschleunigen. Während Wilfarth im Anfange beim Kupferoxyd (resp. Kupfersulfat) blieb, gab er später dem Quecksilberoxyd (resp. Quecksilber) den Vorzug, weil das letztere noch bedeutender die Säurewirkung verstärkt.

Er fand, dass die beschleunigende Wirkung wesentlich von der Löslichkeit in der Säure abhängig ist und dass durch den Zusatz von Phosphorsäureanhydrid zur Sehwefelsäure die Löslichkeit und mit ihr auch die beschleunigende Wirkung des Quecksilbers anf die Oxydation gesteigert wird. Auch fand er - was aber Pflüger und B ohland2) vor ihm für den Harn nachgegewiesen -, dass die nachträgliche Anwendung von Kaliumhypermanganat überflüssig ist, wenn nur die Erwärmung der Säure eine energische ist.

Da durch die sehr bedeutende Verkürzung der zur Analyse nothwendigen Zeit die Methode ausserordentliche praktische Vorzüge bot und besonders zu Stickstoffversuchen, die eine grosse Zahl von genauen Analysen beanspruchen, sich eignete, so habe

1) Chemisches Centralblatt (3. F.) 16, pag. 17 und 113.

2) Dies Archiv Bd. 35, pag. 454; Bd. 36, pag. 102. 
ich mich längere Zeit mit derselben beschäftigt und nachdem ich durch viele Analysen der chemisch reinen Körper der Fett- und aromatischen Reihe mich von der Zuverlässigkeit und der grossen Genauigkeit der Methode überzengte, war ich bemüht, ein möglichst einfaches und ganz gleichmässiges Verfahren zu deren Ausführung mir anzueignen.

In den nachfolgenden Zeilen werde ich, mich möglichst knapp fassend, das Verfahren beschreiben, das sich nach vielen Vorund Fehlversuchen als ein praktisches bewährt hat, wobei ich ausdricklich hervorbebe, dass dasselbe nur auf Combination von verschiedenen längst bekannten und sonst in ihren einzelnen Theilen von anderen befolgten Verfahrungsweisen und Manipulationen beruht. Nichtsdestoweniger glaube ich aber, dass das Verfahren, namentlich was die Destillation betrifft, wesentliche Vortheile bietet und, wie ich hoffe, ziemlich gut den Anforderungen eines Stoffwechselversuchs angepasst ist. Wie gross aber die Anforderungen sind, die ein am Menschen durchgefübrter Stoffwechselversuch an die analytische Methode stellt, siebt man z. B. daraus, dass ich auch bei diesem Verfahren, welches die ganze Analyse in 2 bis 3 Stunden auszuführen gestattet, für einen jeden anderthalbwöchentlichen Stoffwechselversuch doch fast einen ganzen Monat habe analysiren müssen.

$\mathrm{Zu}$ der Beschreibung des von mir befolgten Verfahrens iibergehend, beginne ich mit

\section{der Oxydation.}

Zu der Oxydation nahm ich entweder die reine englische Schwefelsäure oder ein Gemisch derselben mit dem Phosphorsäureanhydrid (auf 1 Liter Säure $200 \mathrm{gr} \mathrm{P}_{2} \mathrm{O}_{5}$ ) - und immer metallisches Quecksilber. Das Quecksilber wurde aus einer 2 cem fassenden engen Bürette jedesmal $\varkappa u \quad 0,1 \mathrm{~cm}$ genommen, entsprechend circa 1,3 gr. Die $\mathrm{H}_{2} \mathrm{SO}_{4}$, resp. die Mischnng der $\mathrm{H}_{2} \mathrm{SO}_{4}$ und $\mathrm{P}_{2} \mathrm{O}_{5}$ („die Sänremischung"), wurde immer in einer und derselben Quantität - ein kleines Probirröhrehen voll, gegen $25 \mathrm{ccm}$ genommen.

Wenn man bei leicht zerlegbaren Substanzen, wie Harn, auch mit der $\mathrm{H}_{2} \mathrm{SO}_{4}$ rasch zum Ziele kommt, so ist es doch fiir die schwerer zersetzlichen, wie z. B. Fleisch, Faeces, Benzolverbindungen ete. rathsamer, das Säuregemisch $\left(\mathrm{H}_{2} \mathrm{SO}_{4}+\mathrm{P}_{2} \mathrm{O}_{5}\right)$ 
anzuwenden, da dadurch eine grosse Zeitersparniss sich ergiebt und ein absolut ruhiges Kochen bei der Oxydation auch bei der heftigsten Flamme erzielt wird. Während z. B. allein mit $\mathrm{H}_{2} \mathrm{SO}_{4}+\mathrm{Hg}$ der Harn $3 / 4-1$ Stunde, Faeces, Fleiseh $21 / 2-3$ Stunden und manche Benzolverbindungen noch etwas mehr Zeit zur vollständigen Entfärbung beanspruchen, so sind bei der Gegenwart auch von $\mathrm{P}_{2} \mathrm{O}_{5}$ der Harn in weniger als $1 / 2$ Stunde, das Fleisch, die Faeces etc. in $1-1 \frac{1}{2}$ Stunden vollständig entfärbt.

Die Oxydation wird in dem üblichen runden langhalsigen Kölbchen (Inhalt $200 \mathrm{cem}$, Halslänge $15 \mathrm{~cm}$ ) vorgenommen, das auf einem Dreifuss über einem Drahtnetz in geneigter Lage erwärmt wird. Erst wird eine schwache Flamme angezindet, nach der Auflösung des Quecksilbers dieselbe verstärkt und bald darauf zur vollen Flamme iibergegangen. Ein Theil des Quecksilbersulfats bleibt in der Lösung, der andere Theil aber bildet einen krystallinischen Niederschlag, der ganz besonders bei Gegenwart von $\mathrm{P}_{2} \mathrm{O}_{5}$ ein absolut ruhiges Kochen bedingt, weshalb irgend welche Vorrichtungen, um sich vor dem Verspritzen der Säure zu schiitzen, absolut unnöthig sind.

Nach der Erreichung einer vollständigen Entfärbung liess ich gewöhulich noch eine $1 / 4$ Stunde kochen und bekam dabei ganz dieselben Resultate, als wenn ich noch mehrere Stunden nach der Entfärbung weiter gekocht hätte.

Während Kjeldahl nur eine Erwärmang bis nabe der Kochtemperatur angegeben, gelangten bekanntlich viele seiner Nachfolger zu der Ueberzeugung, dass die Oxydation einer intensiveren Erwärmung bedarf. Ich bringe das Säuregemisch (resp. die $\mathrm{H}_{2} \mathrm{SO}_{4}$ ) bald nach der Auflösung des Quecksilbers immer in intensives Kochen und unterhalte starkes Kochen bis zum Ende. Die schiefe Stellung des Kölbchens, der lange und enge Kölbchenhals und ein ausserordentlich -gleichmässiges Kochen - auch dann, wenn es intensiv kocht - sehützen vor dem Verlust durch Spritzen vollständig.

Nach dem vollständigen Erkalten des Kölbchens wird vorsichtig erst in dünnem Strahl und in kleinen Quantitäten das Wasser aus einer Spritzflasche zugesetzt, dann das Kölbchen bis etwa $3 / 4$ voll mit Wasser aufgefiullt und durch Schwenken und Drehbewegungen die Flüssigkeiten im Kölbchen zusammengemischt, wobei zugleich mit der Erwärmung der Lösung das Quecksilber- 
salz vollständig sich auflöst und auch nach dem Erkalten der nun verdünnten Säure in Lösung bleibt. Nachdem der wasserklare Inhalt des kleinen Kölbchens mittelst eines Trichters in einen langhalsigen grossen runden Kolben („Destillationskolben“) uibergegossen und das kleine Kölbchen einige Mal nachgespultt, wird zur Destillation ibbergegangen.

\section{Die Destillation.}

Der äusserst einfache Destillationsapparat besteht, wie die Abbildung zeigt, aus 1) einem langhalsigen runden Destillationskolben, 2) einem Schlangenkühler ${ }^{1}$ ) und 3) einer U-förmigen Vorlage (= die Peligot'sche Röbre).

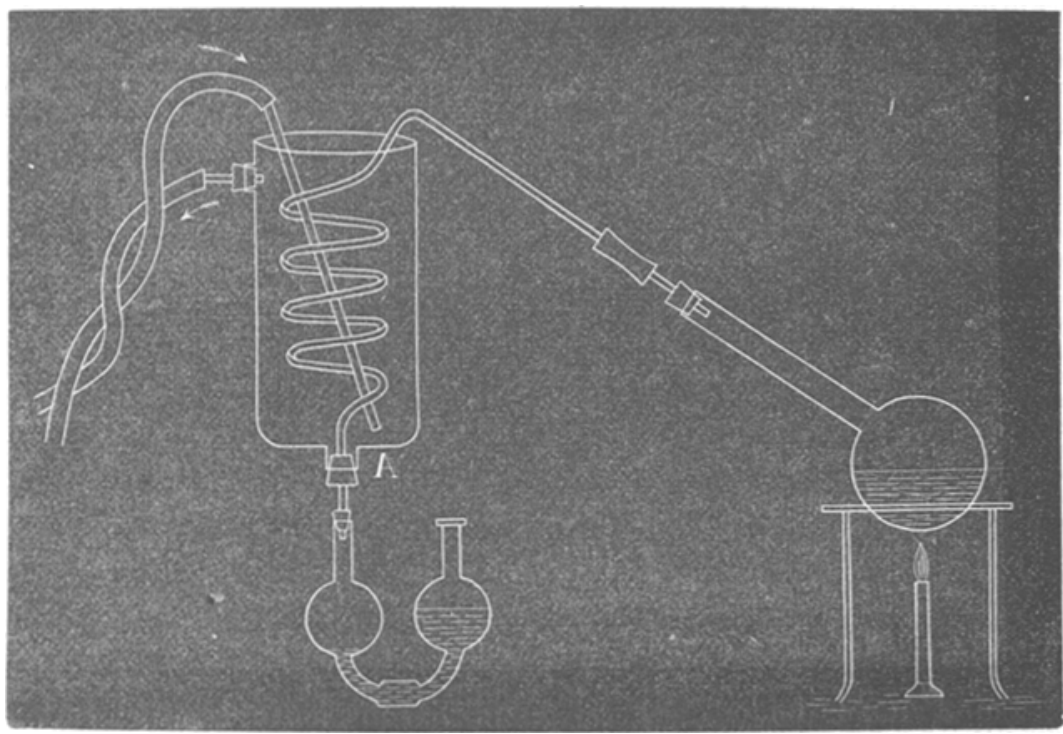

Das Sehlangenrohr des Kühlers ist aus böhmischem, kein Alkali abgebendem Glas so gefertigt, dass es sich oben woch in einen geraden seitlich abgehenden Theil („der ansteigende Theil") abbiegt ${ }^{2}$ ), welcher letztere, gelinde absteigend, ein paar Querfingerbreit oberhalb seines freien, peripherischen Endes 'eine Unterbrechung (von etwa $1 \mathrm{~cm}$ ) erleidet, welche von einem kurzen

1) Der Kolben hat einen Durchmesser von $13^{1 / 2} \mathrm{~cm}$ (fasst $1200 \mathrm{ccm}$ ); der Hals desselben ist $20 \mathrm{~cm}$ lang und $21 / 2 \mathrm{~cm}$ weit.

2) $40 \mathrm{~cm}$ lang. 
Ueber die Kjeldahl-Wilfarth'sche Methode der Stickstoff'bestimmung etc. 585

aber dicken Gummirohr iberbrïckt wird (siehe die Zeichnung). Das freie, peripherische Ende des „ansteigenden Theils" des Kühlrohrs trägt einen einfach durchbohrten Gummistopfen, an dem der Hals des grossen Kolbens bei der Destillation befestigt wird, indem dabei der Kolben auf ein seiner Grösse entsprechend gebogenes auf einem Dreifuss ruhendes Drahtnetz in schräger Stellung zu liegen kommt.

Die U-förmige Peligot'sche Röhre (= die Vorlage), auch zu friberen Zeiten zu Ammoniakbestimmungen als Vorlage benutzt, hat den grossen Vortheil, dass selbst bei einer einzigen Vorlage ein auch minimaler Verlust an Ammoniak kaum möglich ist. An dem unteren senkrechten Theil des Kühlrohrs befestigt, wird die Vorlage bei der Destillation noch in geeigneter Weise durch untergelegte Holzplatten unterstitzt und der mit dem Kühler nicht verbundene. Schenkel der Vorlage vorsichtshalber mit einem Ubrschälchen bedeckt.

Die beschriebene Zusammenstellung und Anordnung des Destillationsapparates hat manche Vortheile zur Folge. Erstens, wenn das Ueberschäumen der kochenden Lauge - welch letzteres nur bei einer fehlerhaften Ausführung der Destillation geschehen kann -- vermieden ist, so können auch bei lange andauerndern Kochen unmöglich die Laugentheilchen in die Vorlage gelangen. Es schuitzt davor erstens die sehiefe Lage des Kolbens, dann der lange enge Hals desselben und endlich der lange ansteigende Theil des Kuhlrohrs - wesshalb noch weitere besondere Vorrichtungen zu diesem Zweck durchaus iiberflüssig werden. Zweitens dauert die Destillation, wegen der geringen Dimensionen des Destillationsapparats und wegen der verbältnissmässig geringen Menge der zu destillirenden Flïssigkeit, eine ziemlich kurze Zeit.

Was endlich die Vorlage betrifft, so schliesst dieselbe mit der titrirten Säure darin den Destillationsraum vollständig von der Aussenluft ab und lässt jedes austretende Bläschen erst die ganze Schicht der Säure hindurehpassiren ${ }^{1}$ ).

1) Die Capacität der Vorlage ist derart gewẩhlt, dass in einer Kugel derselben das gesammte Destillat von 150-200 ccm Platz finden kann. - Es sei hier auch bemerkt, dass die kurze Unterbrechung des geraden ansteigenden Theils des Kühlrohrs nur das leichtere Anbringen und Abnehmen des Kolbens zum Zweck hat. 
Während somit dureh die Einrichtung dieses einfachen Apparates sowohl Alkaliübertritt in die Vorlage, als der Ammoniakverlust mit Sicherheit vermieden werden, hat man bei der Ausführung der Destillation nur für das Erzielen eines möglichst ruhigen Kochens der ziemlich conceptrirten alkalischen Flïssigkeit zu sorgen. Dieses erreicht man 1) durcb die Vermeidung eines grossen Ueberschusses des freien Alkalis und 2) durch Zusatz von geringen Mengen Talk - durch Ausprobiren zu bestimmen zu der zu destillirenden Flüssigkeit. ( $Z$ u viel Talk ist unerwünscht wegen des dabei auftretenden Schäumens.)

Um den Ueberschuss des freien Alkalis in der destillirenden Flüssigkeit jedesmal sehr einzuschränken und dabei doch stets einen unabänderlichen, gleichmässigen Gang der Analyse beizubehalten, verfahre ich folgendermaassen: Nachdem ich eine starke Lauge hergestellt habe ${ }^{1}$ ), bestimme ich erst, wie viel von dieser Lauge zu der gewöhnlich zur Oxydation genommenen Säuremenge eben bis zum Eintritt der alkalischen Reaction zugesetzt werden muss. Dann setze ich die so bestimmte Quantität Lauge welche also jedesmal sicher genügt, die saure Flüssigkeit im Destillationskolben alkalisch zu machen - vor jeder Destillation in der unten beschriebenen Weise der sauren Fliissigkeit zu.

Da die Oxydation unter Zusatz von Quecksilber geschehen, so ist es selbstverständlich, dass in der alkalischen zu destillirenden Flüssigkeit das Ammoniak mit dem Quecksilber zu Mercuramidverbindungen sich vereinigt findet; weil aber diese Verbindungen das Ammoniak schwer abgeben, so hat $W$ ilfarth vorgeschlagen, zu der alkalischen Flüssigkeit unmittelbar vor dem Beginn der Destillation Schwefelkaliumlösung im Ueberschuss zuzusetzen und erst dann zu destilliren. Ich nehme stets $12 \mathrm{ce}$ einer recht concentrirten Schwefelkaliumlösung ${ }^{2}$ ) und setze dieselben zur alkalischen Fliussigkeit unmittelbar vor dem Anbringen des Kolbens an den ansteigenden Theil des Kihlers.

Die Ausführung der Destillation ist folgende:

Es wird aus stets einer und derselben F'resenius'schen (mit einem Glashahn versehenen) Pipette ${ }^{3}$ ) erst $50 \mathrm{ccm}$ der titrirten Schwe-

1) Kalilauge vom spec. Gew. 1,25, Natronlauge vom spec. Gew. 1,25.

2) $1 \mathrm{Th}$. in $1 \frac{1}{2} \mathrm{Th}$. Wasser.

3) Fresenius, Quant. Anal., 6. Aufl., Bd. 1, pag. 38. 
Ueber die Kjeldahl-Wilfarth'sche Methode der Stickstoffbestimmung etc. 587

felsäure in die Vorlage eingegossen und die Vorlage fest an den Kühler angebracht. Dann, nachdem wie schon oben angegeben, aus dem Verbrennungskölbchen die wasserklare Flüssigkeit in den grossen Kolben ubergeführt worden, wird zu derselben etwas Talk (ein kleines Porzellantiegelchen voll) zugesetzt und dann circa $1 / 2$ bis $2 / 3$ der bereits abgemessenen Lauge aus einem Trichter zugegossen, durch Schuittel- und Drehbewegungen alles gut zusammengemischt und einige Minuten zum Abkühlen stehen gelassen, wobei es von Zeit zn Zeit immer tüchtig geschiittelt wird, um den Talk gut in der Flüssigkeit zu vertheilen. Nun wird, indem man unmittelbar am Destillationsapparat steht, aus einem Trichter der Rest der abgemessenen Lauge in den Kolben gegossen, dann der Kolben geschüttelt, die $12 \mathrm{ccm}$ Schwefelkaliumlösung zugesetzt, abermals gesehuittelt und sogleich der Kolben in geneigter Lage fest mit dem Kübler verbunden.

Wenn man genau so verfährt, so braucht man den Verlust des Ammoniaks vor dem Beginn der Destillation durchaus nicht zu befürchten. Da beim angegebenen Verfahren die Fliussigkeit im Kolben beim Zusatz des Restes der abgemessenen Lauge sich nur sehr wenig erwärmt, so kann in den paar Sekunden, die bis zum Anbringen des Kolbens an den Kühler verfliessen, trotz der nun alkalischen Reaction des Kolbeninhaltes ${ }^{1}$ ) doch kein Ammoniakverlust stattfinden, - davon habe ich mich durch vielfache Controlversuche iuberzengt.

Der auf dem Drahtnetze liegende Kolben wird zur Destillation erst durch eine schwache Flamme erwärmt, dann, nachdem Kochen eingetreten, ganz stark erhitzt. Die Destillation dauert bei guter Flamme etwa $3 / 4$ Stunden, wobei eirea $150-200 \mathrm{ccm}$ Flüssigkeit abdestillirt werden, was bei der geringen Menge der Fliissigkeit in dem Destillationskolben vollständig genügt, um alles Ammoniak zu gewinnen.

Vor dem Auslöschen der Flamme unter dem Destillationskolben muss am Ende der Destillation selbstverständlich immer erst die Vorlage von dem Kiuhler abgenommen werden, sonst kann beim Erkalten des Kolbens sehr leicht der Inhalt der mehr weniger gefüllten Vorlage zum Theil zuriuckgesaugt werden.

1) Das Auftreten der alkalischen Reaction im Kolben erkennt man an der Farbenveränderung des Inhalts, an dem Auftreten einer auch beim Schütteln bleibenden Orangefärbung. 
Da die Destillation bei guter Flamme, wie erwähnt, nur circa $3 / 4$ Stunden dauert, so kann man an einem Tage in aller Ruhe bis 8 Destillationen mit einem Apparat vornehmen (bei vielen entsprechend mehr) und weil dabei sowohl oxydirt als titrirt werden kann, so ist es möglich, mit einem Destillationsapparat und einer Vorrichtung zum Oxydiren bis 8 Analysen täglich auszuftibren.

Es braucht auf die Oxydation durchaus nicht sogleich die Destillation zu folgen. Im Gegentheil, dieselbe kann auf eine unbestimmte Zeit aufgeschoben werden ohne im Geringsten die Resultate der Analyse zu beeinflussen. Dies geschieht folgendermassen. Auf eine grosse flache Schïssel mit einer ein paar Centimeter hohen Sehicht von verdünnter Schwefelsäure stellt man eine hohe und flache Glasschale und legt in die letztere eine beliebige Menge von Verbrennungskölbchen; man bedeckt nun diese Schale mit einer grösseren Glasglocke, so dass die Glocke in die verdünnte Schwefelsäure taucht und auf der Schüssel ruht. Auf diese Weise schliesst man die Kölbchen sammt ihrem Inhalte von der Aussenluft durch eine Schicht von verdunnter Schwefelsäure ab, wessbalb kein Ammoniak aus der Luft in die Kölbchen gelangen kann. Es ist klar, dass in eben derselben Weise auch die zur Oxydation vollständig vorbereiteten Kölbchen unbestimmt lange aufbewahrt werden können. Unter 6 Glocken kounte ich anf die Weise 70-80 Kölbchen für spätere Analysen unterbringen.

\section{Die Titration.}

Nach der Ueberführung aus der Vorlage in ein Becherglas nahm ich die Titration des Schwefelwasserstoff enthaltenden Destillats mit einer verdïnnten Kaliumhydratlösung (am besten in der Stärke von 1 bis 2 Zehntel normal) und mit Cochenille als Indicator vor. Bekanntlich liefert die Cochenille auch bei Gegenwart von $\mathrm{H}_{2} \mathrm{~S}$ genaue Resultate. Ich habe ausnahmslos mit Alkali titrirt und in den seltenen Fällen, in denen das Destillat alkalisch war, erst noch einmal 50 ce Säure zugesetzt und dann wie sonst mit Alkali titrirt.

DieCochenilletinctur wurde durch Stehenlassen von $3 \mathrm{gr}$ Cochenille mit 250 ec eines schwachen Spiritus (1 Theil Alkohol auf 3-4 Theile Wasser) bereitet; nach einigen Tagen konnte die anfangs tribe Flitssigkeit klar abgegossen werden. Dieselbe hält 
Ueber die Kjeldahl-Wilfarth'sche Methode der Stickstoffbestimmung etc. $\mathbf{5 8 9}$

sich Monate lang. Von dieser Flïssigkeit habe ich zur Titration immer 20 Tropfen genommen. Als Endreaction ist das Verschwinden der gelben Farbe und das Auftreten der Rosafärbung ohne jede Spur von gelber Nuance anzusehen. Bei einiger Uebung kann man kaum mehr als um 2-3 Tropfen einer 1/10 normalen Kalilösung im Zweifel sein. Schliesslich sei hier noch gesagt, dass die Ausfiuhrung der Titration nach der Destillation, wegen des im Destillat enthaltenen Schwefelwasserstoffs, um durchaus zuverlässig zu sein, kaum viel später als 1 Tag nach der Destillation geschehen darf.

Die unten angeführten Analysen beweisen, dass die Bestimmungsfehler der $\mathrm{Kj}$ jedahl-Wil far th'schen Methode in recht engen Grenzen sich bewegen und beim sorgsamen Arbeiten nur bis 3-6 Decimilligr. betragen, wesshalb diese Methode den sorgfältigsten Stoffwechselversuchen vollkommen genügt und gewiss als eine sehr genaue genannt werden muss.

Die Schärfe dieser Methode hängt wesentlich von der Schärfe der Titration, die eben, um practisch und leicht ausführbar zu bleiben, nicht weiter gesteigert werden kann. Damit hängt es anch zusammen, dass hierbei die Bestimmungsfehler dieselben bleiben, sei es, dass man den Stickstoff erst in Ammoniak uberführen muss, oder sei es, dass das Ammoniak direct aus einem Ammonsalze durch Destillation und Titration gewonnen wird.

$\mathrm{Zu}$ der Untersuchung der Nahrungsmittel und der Excrete tubergehend habe ich folgendes zu berichten. Der Harn wurde stets zu $5 \mathrm{ccm}$ genommen und immer aus einer und derselben engen Bürette in das Verbrennungskölbchen gleichmässig eingetröpfelt.

Die Fäces einer jeden Ausleerung, mit circa $200 \mathrm{ccm}$ einer schwachen Schwefelsäure $\left(10 \mathrm{ccm} \mathrm{H}_{2} \mathrm{SO}_{4}\right.$ auf 1 Liter $\left.\mathrm{H}_{2} \mathrm{O}\right)$ übergossen, wurden später auf dem Wasserbade unter häufigem Umrïhren zu ziemlich gleichmässigem, dicklichen Brei eingedampft und schliess. lich zu einer gleichmässigen asphaltartigen schwarzen Masse eingetrocknet. Dieselben wurden in ein gut verschliessbares Gefäss darauf gebracht, und sofort oder meistens später zu Analysen verwendet.

Von den flïssigen Nahrungsmitteln wurde nur die Milch direct E. Pfüger, Archiv f. Physiologie, Bd. XLVI. 
ans einer Bürette abgemessen, während Bier resp. Wein erst unter dem Zusatz einiger Tropfen $\mathrm{H}_{2} \mathrm{SO}_{4}$ auf dem Wasserbade eingeengt wurden und dann ebenfalls zu $5 \mathrm{ccm}$ zur Analyse genommen. Wenn die eingeengte Flüssigkeit einen Bodensatz enthielt, so wurde sowohl der gesammte Bodensatz als ein Theil der klar abfiltrirten Flïssigkeit besonders analysirt.

Von festen resp. breiigen Nahrungsmitteln wurden nur die Butter und die condensirte Milch mit einem kleinen Blättchen Pergamentpapier von einem bestimmten sehr geringen N-Gehalte oxydirt. Es wurde zuerst das Pergamentpapier und Wägegläschen gewogen, dann in das letztere die Butter resp. cond. Milch hineingethan, abermals gewogen und die Butter, im Papier eingewickelt, in das Verbrennungskölbchen geworfen. (Das Papierblättchen enthielt weniger als $1 \frac{1}{2}$ Decimilligr. N, d. h. 13 solcher Blättchen enthielten zusammen 1,8 milligr. Stickstoff.) Alle anderen Nahrungsmittel wurden ohne jede Zugabe analysirt; sogar der Fleischbrei (resp. Fleischstückchen) lässt sich sehr gut für sich allein aus einem Wägegläschen in das Verbrennungskölbchen überführen.

Die pulverförmigen Substanzen, wie Mehl, gestossener Zwieback etc. wurden in einem langen gedeckten Glasröhrchen gewogen und dasselbe tief bis zur Kolbenkugel zur Ueberführung der betreffenden Substanz eingefubrt.

Die Oxydation einiger eiweiss- und fettreicher Substanzen, so des Fleisches und ganz besonders der Butter, verlangt besondere Vorsicht; es muss nämlich die Flamme beim Beginne der Oxydation so klein, wie nur möglich gemacht werden, weil diese Substanzen im Anfange der Verbrennung ausserordentlich leicht in so starkes Schäumen gerathen, dass sie geradezu überlaufen; sind aber diese Substanzen einige Zeit nur leicht erwärmt worden, dann kann man dieselben ganz dreist und wie sonst zum starken Kochen erhitzen, obne die geringste Gefahr vor dem Ueberlaufen.

Die meiste Zeit zur Entfärbung (bei der Oxydation) brauchen bei gleicbgenommenen Mengen die Butter, die Faeces, das Fleisch; dann kommen die Kohlehydrate. Bei allen eben genannten Substanzen ist in Gegenwart von $\mathrm{P}_{2} \mathrm{O}_{5}$ bei guter Flamme in $1 \frac{1}{2}-2 \frac{1}{2}$ die Oxydation ausnahmslos beendet, ja in den meisten Fällen in 1-1/1/2 Stunden die vollständige Entfärbung erreicht.

Fs ist durchschnittlich vollständig gentigend gegen $1-1,5 \mathrm{gr}$ 
Ueber die Kjeldaht-Wilfarth'sche Methode der Stickstoffbestimmung etc. 591 von den festen, resp. breiigen Nahrungsmitteln zur Analyse zu nehmen. Nur beim Fleischbrei (resp. Fleisch) empfiehlt sich, um möglichst sichere Durchschnittszahlen zu haben, etwas mehr zu nehmen.

Bei allen so eben genannten Substanzen ist das Säuregemisch + $\mathrm{Hg}$ ganz entschieden der reinen $\mathrm{H}_{2} \mathrm{SO}_{4}+\mathrm{Hg}$ vorzuziehen; es geht nicht allein die Verbrennung viel rascher und ruhiger, sondern auch die Destillation geht hiernach besser vor sich, da das Säuregemisch nicht zu lange gekocht zu werden braucht und daher nicht viel Säure verdampft.

Diese Arbeit ist zum Theil in St. Petersburg in dem chemischen Laboratorium des Forst-Instituts, zum Theil im Bonner physiologischen Institut gemacht. Ich hatte im vorigen Jahre (1888) eine Arbeit über die Kjedahl-Wilfarth'sche Methode in russischer Sprache publicirt, seitdem aber das in jener Arbeit beschriebene Verfahren modificirt und vereinfacht.

Analytische Belege.

\begin{tabular}{|c|c|c|c|c|c|c|c|}
\hline \multirow{2}{*}{$\begin{array}{l}\text { Analysirte } \\
\text { Substanz }\end{array}$} & \multirow{2}{*}{$\begin{array}{l}\text { Gewicht } \\
\text { in } \\
\text { Gramm. }\end{array}$} & \multicolumn{3}{|c|}{ Milligramme Stickstoff } & \multicolumn{3}{|c|}{ Procente Stickstoff } \\
\hline & & $\begin{array}{c}\text { be- } \\
\text { rechnet }\end{array}$ & $\begin{array}{c}\text { ge- } \\
\text { funder }\end{array}$ & $\left|\begin{array}{c}\text { Diffe- } \\
\text { renz }\end{array}\right|$ & $\begin{array}{c}\text { be- } \\
\text { rechnet }\end{array}$ & $\begin{array}{c}\text { ge- } \\
\text { funden }\end{array}$ & $\begin{array}{l}\text { Diffe- } \\
\text { renz }\end{array}$ \\
\hline Benzidin & $\begin{array}{l}0,2724 \\
0,2352 \\
0,2909 \\
0,2911 \\
0,3090\end{array}$ & $\begin{array}{l}41,45 \\
35,79 \\
44,27 \\
44,30 \\
47,02\end{array}$ & $\begin{array}{l}41,19 \\
35,74 \\
44,20 \\
44,20 \\
46,72\end{array}$ & $\begin{array}{r}-0,26 \\
-0,05 \\
-0,07 \\
-0,10 \\
-0,30\end{array}$ & $\begin{array}{c}15,22 \\
" \\
" \\
"\end{array}$ & $\begin{array}{l}15,12 \\
15,20 \\
15,20 \\
15,19 \\
15,12\end{array}$ & $\begin{array}{r}-0,10 \\
-0,02 \\
-0,02 \\
-0,03 \\
-0,10\end{array}$ \\
\hline Harnstoff & & $\begin{array}{r}101,88 \\
75,18 \\
78,96 \\
60,60\end{array}$ & $\begin{array}{r}101,86 \\
74,91 \\
78,78 \\
60,67\end{array}$ & $\begin{array}{l}+0,03 \\
-0,27 \\
-0,18 \\
+0,07\end{array}$ & $\begin{array}{c}46,67 \\
" \\
" \\
n\end{array}$ & $\begin{array}{l}46,68 \\
46,50 \\
46,56 \\
46,70\end{array}$ & $\begin{array}{r}+0,01 \\
-0,17 \\
-0,11 \\
+0,03\end{array}$ \\
\hline Hipursäure & $\begin{array}{l}0,2696 \\
0,2517 \\
0,2473 \\
0,1841\end{array}$ & $\begin{array}{l}21,1 \\
19,7 \\
19,3 \\
14,4\end{array}$ & $\begin{array}{l}21,4 \\
20,2 \\
19,8 \\
14,8\end{array}$ & $\begin{array}{l}+0,3 \\
+0,5 \\
+0,5 \\
+0,4\end{array}$ & $\begin{array}{l}7,82 \\
" \\
" \\
"\end{array}$ & $\begin{array}{l}7,94 \\
8,02 \\
8,02 \\
8,03\end{array}$ & $\begin{array}{l}+0,12 \\
+0,20 \\
+0,20 \\
+0,21\end{array}$ \\
\hline Benzoiltoluidin & 0,3154 & 20,9 & 20,5 & $-0,4$ & 6,63 & 6,51 & $-0,12$ \\
\hline Tyrosin & 0,2279 & 17,6 & 18,0 & $+0,4$ & 7,73 & 7,93 & $+0,20$ \\
\hline Leucin & 0,1297 & 13,9 & 14,0 & $+0,1$ & 10,69 & 10,78 & $+0,09$ \\
\hline Acetanilid & 0,4004 & 41,5 & 41,7 & $+0,2$ & 10,37 & 10,42 & $+0,05$ \\
\hline
\end{tabular}


Nahrungsmittel I (Fleiseb) ${ }^{1}$.

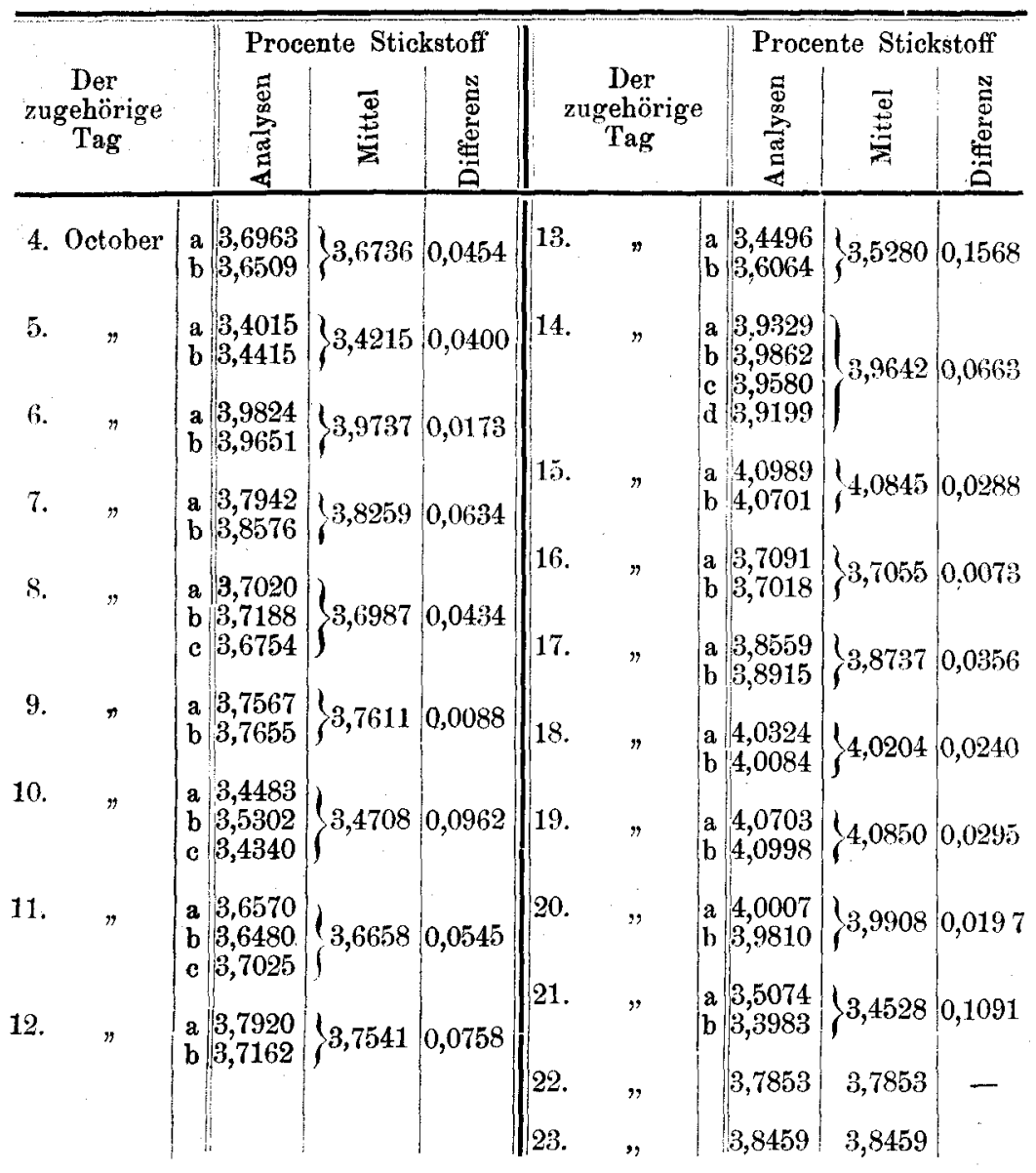

1) Als ein Beispiel dessen, wie weit die verschiedenen Analysen vom Fleisch eines und desselben Tages mit einander übereinstimmen, sind hier die von den Versuchsreihen $\mathrm{C}$ und $\mathrm{D}$ angeführt. 
Ueber die Kjeldahl-Wilfarth'sche Methole der Stickstoffbestimmung etc, 598

Nahrungsmittel II.

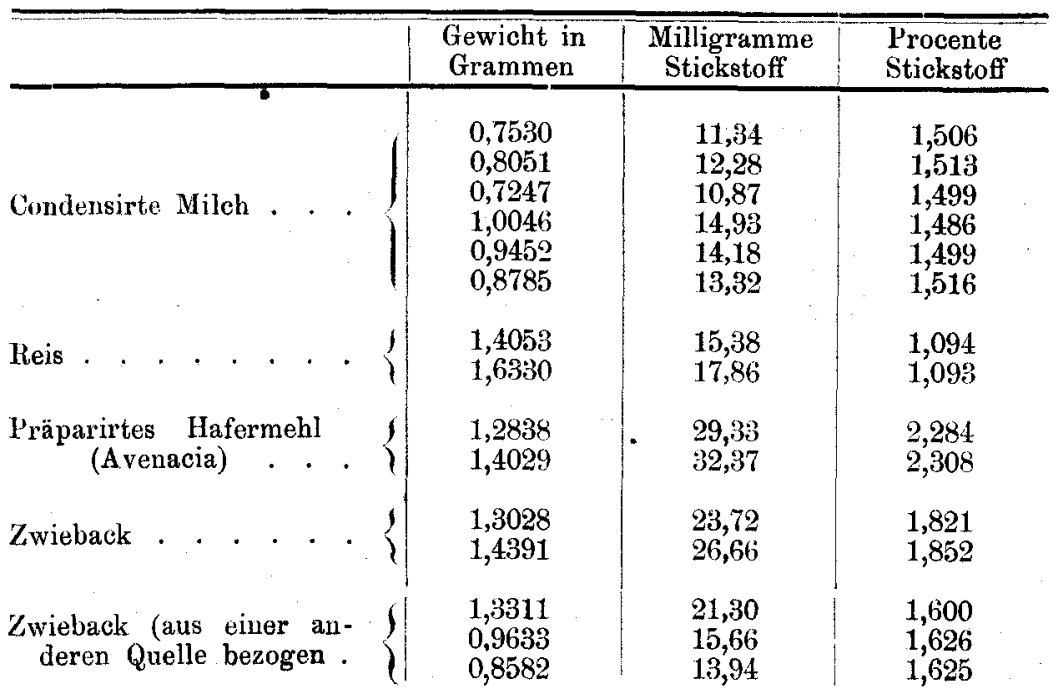

Excrete.

\begin{tabular}{|c|c|c|c|c|c|}
\hline$H$ a $r n$ & $\begin{array}{l}\text { Procente } \\
\text { Stickstoff }\end{array}$ & $\begin{array}{l}\text { Getrocknete } \\
\text { in Gram }\end{array}$ & $\begin{array}{l}\text { Fäces } \\
\text { men }\end{array}$ & $\begin{array}{l}\text { Milligr. } \\
\text { Stickstoff }\end{array}$ & $\begin{array}{l}\text { Procente } \\
\text { Stickstoff }\end{array}$ \\
\hline Vom 11. Oct. $\left\{\begin{array}{c}5 \mathrm{cc} . \\
n\end{array}\right.$ & $\begin{array}{l}0,9066 \\
0,9048 \\
0,9029\end{array}$ & Vom 8. Oct. & $\begin{array}{l}0,6539 \\
0,5628\end{array}$ & $\begin{array}{l}42,54 \\
37,31\end{array}$ & $\begin{array}{l}6,506 \\
6,627\end{array}$ \\
\hline Vom t5.0c.t $\left\{\begin{array}{c}5 \mathrm{cc} . \\
\eta\end{array}\right.$ & $\begin{array}{l}0,6273 \\
0,6244\end{array}$ & Vom 20. Oct. & $\begin{array}{l}0,7593 \\
0,5605\end{array}$ & $\begin{array}{l}41,79 \\
30,39\end{array}$ & $\begin{array}{l}5,504 \\
5,422\end{array}$ \\
\hline Vom 16. Oct. $\left\{\begin{array}{c}5 \mathrm{cc} . \\
n\end{array}\right.$ & $\begin{array}{l}0,6283 \\
0,6283 \\
0,6264\end{array}$ & Vom 25. Oct. & $\begin{array}{l}0,7731 \\
0,6750\end{array}$ & $\begin{array}{l}38,43 \\
34,41\end{array}$ & $\begin{array}{l}4,971 \\
5,097\end{array}$ \\
\hline
\end{tabular}

